\title{
Preliminary study of insects associated to indoor body decay in Colombia ${ }^{1}$
}

\author{
Yardany Ramos-Pastrana ${ }^{2}$, Alexander Velasquez-Valencia ${ }^{2} \&$ Marta Wolff $^{3}$
}

\author{
${ }^{1}$ Contribution from University of Amazonia and University of Antioquia to Forensic Entomology. \\ ${ }^{2}$ Museo de Historia Natural, Universidad de la Amazonia, Grupo Fauna Silvestre, Sede Centro, Carrera 11 No. 6-69 Barrio Juan XIII, Florencia, \\ Caquetá, Colombia.bioyardany@yahoo.com.mx; alexandervelasquezvalencia@gmail.com \\ ${ }^{3}$ Grupo de Entomología, Instituto de Biología, Universidad de Antioquia, A.A. 1226, Medellín, Colombia. martha.wolff@udea.edu.co
}

\begin{abstract}
Preliminary study of insects associated to indoor body decay in Colombia. This is the first report studying insects associated to indoor body decay process of a white pig (Sus scrofa) (Artiodactyla, Suidae) in a controlled indoor environment in an urban area of Florencia city, Amazonia Piedmont, Colombia. For a period of 54 days, 9,220 individuals (immature and adults), distributed in 3 orders, 5 families, 10 genera, and 10 species were collected using entomological nets and tweezers. Five decaying stages are described (fresh, bloated, active decay, advanced decay and remains). During the fresh stage we recorded Cochliomyia macellaria (Fabricius, 1775), Chrysomya albiceps (Wiedemann, 1819), Ophyra aenescens (Wiedemann, 1830), Oxysarcodexia sp., Lepidodexia sp. and Lasiophanes sp.; during the bloating stage C. macellaria, C. albiceps, Lucilia eximia (Wiedemann, 1819), Hemilucillia semidiaphana (Rondani, 1850), Musca domestica Linnaeus, 1758, O. aenescens, Oxysarcodexia sp., Lepidodexia sp., Dermestes maculatus De Geer, 1774 and Lasiphanes sp.; during the active decay C. macellaria, C. albiceps, $L$. eximia, M. domestica, O. aenescens, Lepidodexia sp. D. maculatus and Lasiophanes sp.; during the advanced decay C. macellaria, C. albiceps, M. domestica, Lepidodexia sp. and Lasiophanes sp.; and during the remains stage C. albiceps, D. maculatus and Lasiophanes sp. The insects were sorted out in 3 ecological categories; necrophagous, predators and parasites and sarco-saprophagous. According to Chao and Jack estimators, total richness was observed on day 20 , with $100 \%$ of the expected species.
\end{abstract}

KEYWORDS. Amazon Region; Forensic Entomology; Indoor Closed, Post-Mortem Interval.

Forensic Entomology is the application of the study of insects in cases of legal nature, in any of its three components: urban, stored products and medico-legal (Hall \& Huntington 2010); these use of insects as a tool in legal situations, has been well documented since the thirteenth century and artistically presented by sculptors, painters and poets since Middle Ages (Benecke 2008). Analysis of the distribution, biology and behavior of the insects found in a crime scene yields valuable information about where, when, and sometimes how the facts being investigated took place (Hall et al. 2008). Factors such environmental conditions (temperature, rainfall), clothing, body depth and weight affect the rate of decomposition of the body, consequently insect colonization (Mann et al. 1990).

Studies on arthropods succession associated to outdoor animal remains decomposition are well documented in temperate regions (Anderson \& VanLaerhoven 1996; Arnaldos et al. 2001; Grassberger \& Frank 2004; Tabor et al. 2005; De Jong \& Hoback 2006), as well as for the Neotropical region (Jirón et al. 1982; Moura et al. 1997; Cruz \& Vasconcelos 2006; Carvalho et al. 2000, Carvalho \& Linhares 2001; Iannacone 2003; Velásquez 2008; Souza et al. 2008; Vasconcelos \& Araujo 2012). In Colombia, studies on forensic entomology started out in 2001, and have focused mainly on patterns of decomposition in outdoor environments and aquatics conditions (Wolff et al. 2001; Pérez et al. 2005; Camacho 2005; Martínez et al. 2007; Segura et al. 2009; Grisales et al. 2010; Segura et al. 2011; Ramos-Pastrana \& Wolff 2011; Barrios \& Wolff 2011).

After temperature, access to carcasses by insects is the most important factor affecting the decaying rate (Mann et al. 1990). Studies of fauna in carrion in controlled indoor environments have been restricted around the world, and particularly the great majority has been made directly on human remains, like the one performed in Hawaii in which exclusive species for both open and closed habitats were determined (Goff 1991). That same year, in Gdansk Poland, Piatkowski (1991) analyzed the entomologic fauna of a remain found on the $11^{\text {th }}$ floor of a building; later in Germany, Benecke (1998) and Schroeder et al. (2002) analyzed entomologic evidence of remains found inside apartments. In Argentina, Centeno et al. (2002) carried out the first study in controlled indoor environments using pigs carcass; in the United States, Gennard (2007) analyzed the development of blow flies in bodies found both indoors and outdoors; in Finland, Pohjoismäki et al. (2010) observed the colonization of insects in bodies inside rooms; in Germany, Frost et al. (2010) indicated that $81.9 \%$ of the 364 bodies infested with insects were found indoors; in Malaysia, Ahmad et al. (2011) compared the decaying bodies of monkeys indoors and outdoors.

There are no reports of this kind of studies in Colombia, so this research aims at determining the entomological succession, ecological categories and decaying rates of a body of a white pig (Sus scrofa) in a controlled indoor environment, in Florencia city, Caquetá-Colombia. 


\section{MATERIAL AND METHODS}

The study was carried out in an area of $4 \mathrm{~m}^{2}$, in an old bath located on the seventh floor of a building, $70 \mathrm{~m}$ from the ground. The bathroom has no windows, a door which remained closed, leaving only a space for the entrance of the insects of approximately $2 \mathrm{~cm}$ between the door and the bathroom floor. This building is located in the urban area of Florencia, $\left(2^{\circ} 58^{\prime} \mathrm{N}\right.$ and $0^{\circ} 40^{\prime} \mathrm{S}$ and between $71^{\circ} 30^{\prime}$ and $76^{\circ} 15^{\prime}$ ' W., at $270 \mathrm{~m}$.) with an annual mean rainfall of 3,793 $\mathrm{mm}$, average temperature of $25^{\circ} \mathrm{C}$, mean annual relative humidity of $92 \%$. The region is characterized by being the intersection zone between the piedmont and the Amazonia planes, featured by small hills and alluvial terraces which resulted from accumulation of materials, forming reliefs that go from plane to undulate and abrupt (IGAC 2010).

The study was carried out using a white pig (Sus scrofa) of approximately $14.5 \mathrm{~kg}$, slaughtered at the study site (performed by a professional of "Cuerpo Técnico de Investigación", the Technical Body of Researches C.T.I. for the Spanish acronym, of the Nation General Prosecutors), by means of impact of a caliper $9 \mathrm{~mm}$ bullet in the frontal region of the skull, on July 20, 2010 at approximately 06:00 a.m. From the moment of death until the carcass was only bones and skin, photographs were taken and notes of the physical changes observed were recorded; samples of insects were taken four times every day (06:00, 10:00, 14:00 and 18:00 hours) all along the decaying process. Besides, factors such as body weight, room temperature (thermo-hygrometer digital Thermo) and rectal body temperature, (Elan digital thermometer) were recorded.

Sampling of adult flying insects was made using entomological nets of $20 \mathrm{~cm}$ in diameter; non-flying adults and immature individual found on, under, and around the body were collected using tweezers and fine tip brushes, following the methodology proposed by Haskell (1990). The individuals collected were taxonomically determined to species when possible, using the keys proposed by Mariluis \& Peris (1984); Smith (1986); Queiroz \& Carvalho (1987); Peris (1992); Ribeiro \& Carvalho (1998); Carvalho \& Ribeiro (2000); Palacio \& Fernández (2003); Amat et al. (2008); Almeida \& Mise (2009); Flórez \& Wolff (2009); Brown et al. (2009); Brown et al. (2010); Carvalho et al. (2012).

Life cycles. Once the first masses of eggs were found, they were taken into rearing chambers, consisting of polythene containers with lids, filled with white sterile sand to facilitate pupation. Larvae where fed with ground cow meat, the chambers were kept in styrofoam containers to avoid contamination by other insects and the temperature was kept constant. Rearing chambers were taken into the Entomology lab at the Natural History Museum of Universidad de la Amazonia, where observations and sampling were made in periods of four hours each $(06: 00,10: 00,14: 00$ y 18:00 hours). Five larvae were collected in each sampling period, and stored in vials with alcohol at $75 \%$ for their conservation, and date, hour, temperature, and relative humidity were recorded. Observations were made until adults emerged which were then used for taxonomic determination of the pioneer species.

Data Analysis. This study was designed to evaluate the succession pattern of insects during the decomposition of a cadaver indoors, and was not designed for a quantitative analysis. To determine the differences between decaying stages an analysis of variance was performed and Turkey test $(\mathrm{P}<0.05)$ was applied. To determine if the sampling effort was optimal, a cumulative curve of species was calculated using Chao1, Chao2, Jackknife1 and Jackknife 2 estimators, which are based on the incidence (present-absent) (JiménezValverde \& Hortal 2003), using the software EstimateS version 8.0 for Windows (Colwell 2006). Each decomposition stage was correlated to a group of insects, environmental and physical factors such as room and body temperature, and weight loss. A succession table and an occurrence matrix of presence-absence were built. Graphics showing the physical and environmental conditions during the decaying process were plotted. Ecological categories of each species were used according to Smith (1986) and Magaña (2001).

\section{RESULTS}

A total of 9,220 adults and immature insects were collected, distributed in 3 orders, 5 families, 10 genera and 10 species (Table I). The most abundant order was Diptera with 8,703 individuals (94\%), of which Calliphoridae was the dominant family with 6,878 individuals $(75 \%)$, followed by Muscidae 1,454 (16\%), and Sarcophagidae with 371 (4\%) (Tables II, III). Coleoptera was the second most abundant order, with Dermestidae 301 individuals (3\%); followed by Formicidae (Hymenoptera) 216 (2\%) (Tables II, III).

Decaying stages and associated insects. Total decay lasted 54 days, five decaying stages statistically different $(\mathrm{P}$ $<0.05)$ were identified: Fresh, Bloated, Active, Advanced and Remains.

Fresh (day 0 to 2). Fresh decaying stage started from the moment of decease until the first signs of body bloating were observed. No characteristic odors of decomposition were perceived, lowering of body temperature was evident from $28.75^{\circ} \mathrm{C}$ to $24^{\circ} \mathrm{C}$, while room temperature ranged between $23^{\circ} \mathrm{C}$ and $26^{\circ} \mathrm{C}$, relative humidity oscillated between $67 \%$ and $98 \%$ (Fig. 1). During this stage, body biomass loss was $1.12 \%$ (Fig. 2). Mainly adult individuals of Diptera, Cochliomyia macellaria and Chrysomya albiceps (Calliphoridae), Ophyra aenescens (Muscidae) and Oxysarcodexia sp. (Sarcophagidae) were found, while Lepidodexia sp. (Sarcophagidae) was found at an immature stage; Lasiophanes sp. (Formicidae) was also present (Tables II, III, IV).

Bloated (day 3 to 5). Bloated decaying stage started at the moment when carcass bloating was evident due to the gases originated by anaerobic bacteria. It was the easiest stage to differentiate due to the spherical shape taken by the body. Initially, the abdomen was bloated then the entire body; period in which characteristic odors of decaying ani- 

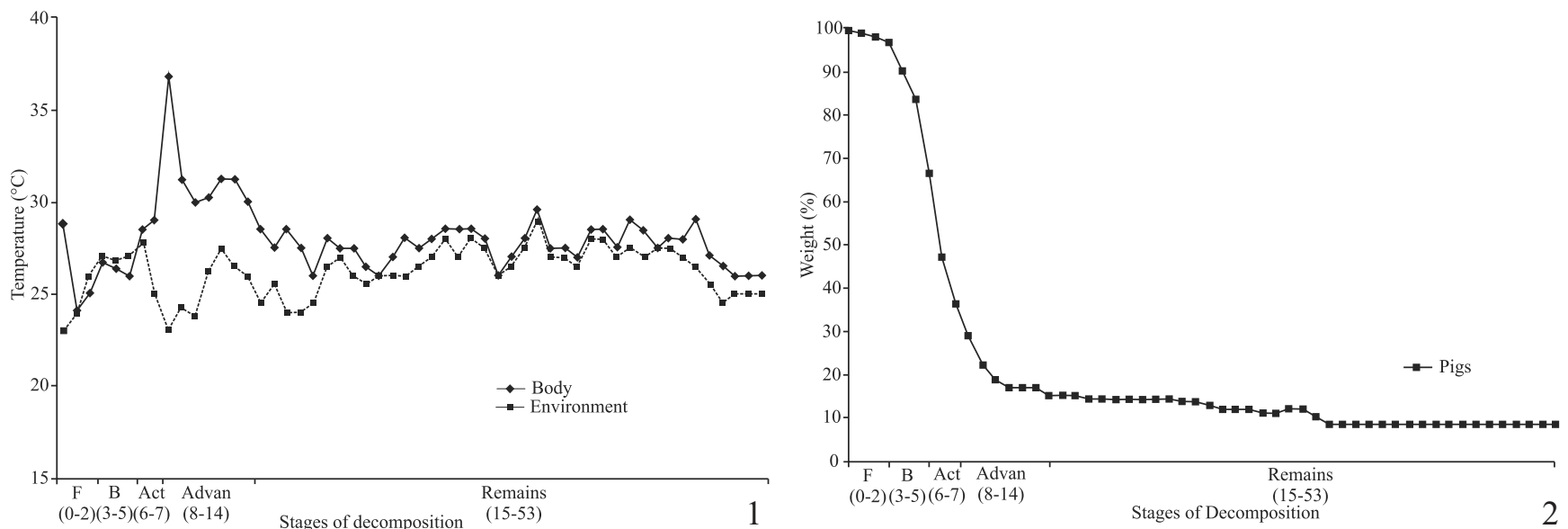

Figs. 1-2. Daily variation of (1) temperature and (2) weight related with the stage of decomposition in a closed precinct of the Colombia Amazonia Piedmont.

Table I. Succession of immature (I) and adult (A) insects in different stages of cadaveric decomposition collected in a closed precinct of the Colombia Amazonia Piedmont. N $=$ necrofagous, $\mathrm{P}=$ parasite, $\mathrm{S}=$ saprofagous

\begin{tabular}{|c|c|c|c|c|c|c|c|c|c|c|c|c|c|}
\hline \multirow{4}{*}{ Order } & \multirow{4}{*}{ Family } & \multirow{4}{*}{ Specie } & \multirow{4}{*}{ Ecol. Cat. } & \multicolumn{10}{|c|}{ Stage } \\
\hline & & & & \multirow{2}{*}{\multicolumn{2}{|c|}{$\begin{array}{c}\text { Fresh } \\
(0-2)\end{array}$}} & \multirow{2}{*}{\multicolumn{2}{|c|}{$\frac{\text { Bloated }}{(3-5)}$}} & \multirow{2}{*}{\multicolumn{2}{|c|}{$\frac{\text { Active }}{(6-7)}$}} & \multirow{2}{*}{\multicolumn{2}{|c|}{$\begin{array}{c}\text { Advanced } \\
(8-14)\end{array}$}} & \multirow{2}{*}{\multicolumn{2}{|c|}{$\frac{\text { Remains }}{(15-53)}$}} \\
\hline & & & & & & & & & & & & & \\
\hline & & & & $\mathrm{I}$ & $\bar{A}$ & $\mathrm{I}$ & $\bar{A}$ & I & $\mathrm{A}$ & I & $\bar{A}$ & I & $\overrightarrow{\mathrm{A}}$ \\
\hline \multirow[t]{8}{*}{ Diptera } & Calliphoridae & Chrysomya albiceps & $\mathrm{N}, \mathrm{P}$ & & $\mathrm{x}$ & $\mathrm{x}$ & $\mathrm{x}$ & $\mathrm{x}$ & $\mathrm{x}$ & $\mathrm{x}$ & $\mathrm{x}$ & $\mathrm{x}$ & $\mathrm{x}$ \\
\hline & & Cochliomyia macellaria & $\mathrm{N}$ & & $\mathrm{x}$ & $\mathrm{x}$ & $\mathrm{x}$ & $\mathrm{x}$ & $\mathrm{x}$ & $\mathrm{x}$ & $\mathrm{x}$ & & \\
\hline & & Lucilia eximia & $\mathrm{N}$ & & & $\mathrm{x}$ & $\mathrm{x}$ & $\mathrm{x}$ & & & & & \\
\hline & & Hemilucilia semidiaphana & $\mathrm{N}$ & & & & $\mathrm{x}$ & & & & & & \\
\hline & Muscidae & Musca domestica & S & & & $\mathrm{x}$ & & $\mathrm{x}$ & & $\mathrm{x}$ & & & \\
\hline & & Ophyra aenescens & S & & $\mathrm{x}$ & & $\mathrm{x}$ & & $\mathrm{x}$ & & & & \\
\hline & Sarcophagidae & Oxysarcodexia sp. & $\mathrm{N}, \mathrm{S}$ & & $\mathrm{x}$ & & $\mathrm{x}$ & & & & & & \\
\hline & & Lepidodexia sp. & $\mathrm{N}, \mathrm{S}$ & $\mathrm{x}$ & & $\mathrm{x}$ & & $\mathrm{x}$ & & $\mathrm{x}$ & & & \\
\hline Coleoptera & Dermestidae & Dermestes maculatus & $\mathrm{N}$ & & & & $\mathrm{x}$ & & $\mathrm{x}$ & $\mathrm{x}$ & $\mathrm{x}$ & $\mathrm{x}$ & $\mathrm{x}$ \\
\hline Hymenoptera & Formicidae & Lasiophanes sp. & $\mathrm{P}$ & & $\mathrm{x}$ & & $\mathrm{x}$ & & $\mathrm{x}$ & & $\mathrm{x}$ & & $x$ \\
\hline
\end{tabular}

Table II. Percentage of adult individual insects associated to a cadaveric decomposition in a closed precinct of the Colombia Amazonia Piedmont.

\begin{tabular}{|c|c|c|c|c|c|c|c|}
\hline Order & Family & Species & Fresh $(0-2)$ & Bloated (3-5) & Active (6-7) & Advanced (8-14) & Remais (15-53) \\
\hline \multirow[t]{8}{*}{ Diptera } & Calliphoridae & Cochliomyia macellaria & $0.15(\mathrm{n}=14)$ & $2.79(\mathrm{n}=257)$ & $0.20(\mathrm{n}=18)$ & $0.03(\mathrm{n}=3)$ & $0(\mathrm{n}=0)$ \\
\hline & & Chrysomya albiceps & $0.02(\mathrm{n}=2)$ & $0.31(\mathrm{n}=28)$ & $0.10(\mathrm{n}=9)$ & $0.21(\mathrm{n}=19)$ & $1.21(\mathrm{n}=112)$ \\
\hline & & Hemilucilia semidiaphana & $0(\mathrm{n}=0)$ & $0.02(\mathrm{n}=2)$ & $0.01(\mathrm{n}=1)$ & $0(\mathrm{n}=0)$ & $0(\mathrm{n}=0)$ \\
\hline & & Lucilia eximia & $0(\mathrm{n}=0)$ & $0(\mathrm{n}=0)$ & $0(\mathrm{n}=0)$ & $0(\mathrm{n}=0)$ & $0(\mathrm{n}=0)$ \\
\hline & Muscidae & Musca domestica & $0(\mathrm{n}=0)$ & $0(\mathrm{n}=0)$ & $0(\mathrm{n}=0)$ & $0(\mathrm{n}=0)$ & $0(\mathrm{n}=0)$ \\
\hline & & Ophyra aenescens & $0.17(\mathrm{n}=16)$ & $3.77(\mathrm{n}=348)$ & $2.22(\mathrm{n}=205)$ & $0.08(\mathrm{n}=7)$ & $0(\mathrm{n}=0)$ \\
\hline & Sarcophagidae & Oxysarcodexia sp. & $0.11(\mathrm{n}=10)$ & $0.03(\mathrm{n}=3)$ & $0(\mathrm{n}=0)$ & $0(\mathrm{n}=0)$ & $0(\mathrm{n}=0)$ \\
\hline & & Lepidodexia sp. & $0(\mathrm{n}=0)$ & $0(\mathrm{n}=0)$ & $0(\mathrm{n}=0)$ & $0(\mathrm{n}=0)$ & $0(\mathrm{n}=0)$ \\
\hline Coleoptera & Dermestidae & Dermestes maculatus & $0(\mathrm{n}=0)$ & $0.03(\mathrm{n}=3)$ & $0.03(\mathrm{n}=3)$ & $0.34(\mathrm{n}=31)$ & $0.34(\mathrm{n}=31)$ \\
\hline Hymenoptera & Formicidae & Lasiophanes sp. & $0.36(\mathrm{n}=33)$ & $0.28(\mathrm{n}=26)$ & $0.15(\mathrm{n}=14)$ & $0.34(\mathrm{n}=31)$ & $1.21(\mathrm{n}=112)$ \\
\hline
\end{tabular}

Table III. Percentage of immature individual insects associated to a cadaveric decomposition in a closed precinct of the Colombia Amazonia Piedmont.

\begin{tabular}{|c|c|c|c|c|c|c|c|}
\hline Order & Family & Species & Fresh $(0-2)$ & Bloated (3-5) & Active (6-7) & Advanced (8-14) & Remains (15-53) \\
\hline \multirow[t]{8}{*}{ Diptera } & Calliphoridae & C. macellaria & $0(\mathrm{n}=0)$ & $0.78(\mathrm{n}=72)$ & $16.87(\mathrm{n}=1555)$ & $4.52(n=417)$ & $0.03(\mathrm{n}=3)$ \\
\hline & & Ch. albiceps & $0(\mathrm{n}=0)$ & $0.31(\mathrm{n}=29)$ & $17.82(\mathrm{n}=1653)$ & $8.80(\mathrm{n}=811)$ & $7.38(\mathrm{n}=680)$ \\
\hline & & H. semidiaphana & $0(\mathrm{n}=0)$ & $0(\mathrm{n}=0)$ & $0(\mathrm{n}=0)$ & $0(\mathrm{n}=0)$ & $0(\mathrm{n}=0)$ \\
\hline & & L. eximia & $0(\mathrm{n}=0)$ & $0.03(\mathrm{n}=3)$ & $0.03(\mathrm{n}=3)$ & $0(\mathrm{n}=0)$ & $0(\mathrm{n}=0)$ \\
\hline & Muscidae & M. domestica & $0(\mathrm{n}=0)$ & $0.01(\mathrm{n}=1)$ & $5.01(\mathrm{n}=462)$ & $4.65(n=429)$ & $0(\mathrm{n}=0)$ \\
\hline & & O. aenescens & $0(\mathrm{n}=0)$ & $0(\mathrm{n}=0)$ & $0(\mathrm{n}=0)$ & $0(\mathrm{n}=0)$ & $0(\mathrm{n}=0)$ \\
\hline & Sarcophagidae & Oxysarcodexia $\mathrm{sp}$. & $0(\mathrm{n}=0)$ & $0(\mathrm{n}=0)$ & $0(\mathrm{n}=0)$ & $0(\mathrm{n}=0)$ & $0(\mathrm{n}=0)$ \\
\hline & & Lepidodexia sp. & $0.52(\mathrm{n}=48)$ & $0.10(\mathrm{n}=9)$ & $1.18(\mathrm{n}=109)$ & $2.07(\mathrm{n}=196)$ & $0(\mathrm{n}=0)$ \\
\hline Coleoptera & Dermestidae & D. maculatus & $0(\mathrm{n}=0)$ & $0(\mathrm{n}=0)$ & $0(\mathrm{n}=0)$ & $0(\mathrm{n}=0)$ & $2.53(\mathrm{n}=233)$ \\
\hline Hymenoptera & Formicidae & Lasiophanes sp. & $0(\mathrm{n}=0)$ & $0(\mathrm{n}=0)$ & $0(\mathrm{n}=0)$ & $0(\mathrm{n}=0)$ & $0(\mathrm{n}=0)$ \\
\hline
\end{tabular}

Revista Brasileira de Entomologia 58(4): 326-332, December 2014 
mal matter were perceived, and rigidity of legs and hardiness of the great muscles became evident. Body temperature was homogeneous, fluctuating between $26^{\circ} \mathrm{C}$ and $26.75^{\circ} \mathrm{C}$, room temperature ranged between $26.75^{\circ} \mathrm{C}$ and $27^{\circ} \mathrm{C}$ and relative humidity oscillated between $75 \%$ and $94 \%$ (Fig. 1). During this stage biomass loss was $7.16 \%$ (Fig. 2). Adult and immature individuals of $C$. macellaria, $C$. albiceps and Lucilia eximia were observed, while Hemilucillia semidiaphana (Calliphoridae), O. aenescens, Oxysarcodexia sp., Lasiophanes sp. and Dermestes maculatus (Coleoptera: Dermestidae) were found only in adult stage; Musca domestica (Muscidae) and Lepidodexia sp. in immature stage (Tables II, III, IV). The first laying of Diptera was observed in natural orifices of the body such as eyes, ears, nose, mouth and anus, and in the wound. A follow up of the life cycle of these eggs led to determine the adult form of C. macellaria (Fig. 3).

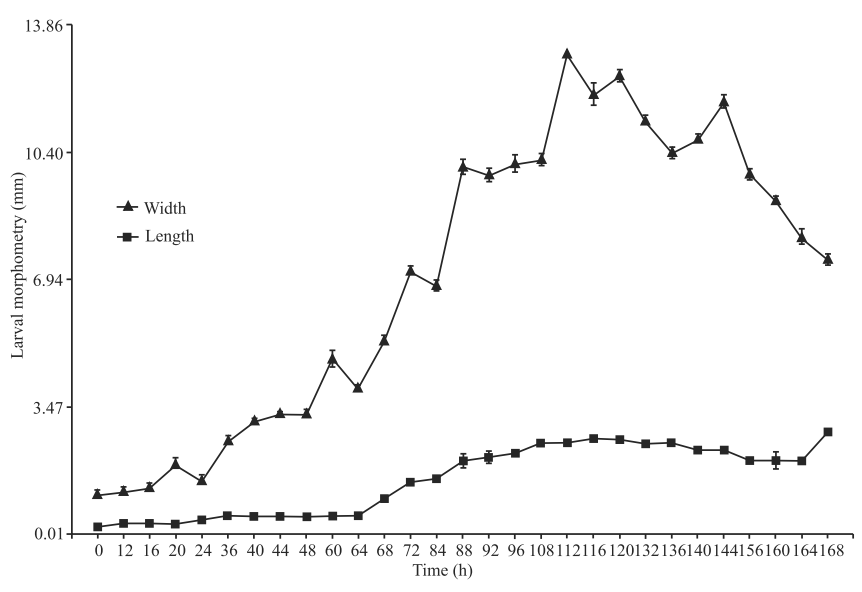

Fig. 3. Average length and width (in $\mathrm{mm}$ ) of larvae of Cochliomyia macellaria during growth in the Colombia Amazonia Piedmont.

Active (day 6 to 7). This stage is characterized by a humid carcass and rupture of the skin, which allows gases to escape, so the body begins to deflate gradually. Natural orifices become less defined and the body starts to lose its original shape still preserving its muscular end epithelial tissues. Rectal body temperature fluctuated between $28.5^{\circ} \mathrm{C}$ and $29^{\circ} \mathrm{C}$, while room temperature between $25^{\circ} \mathrm{C}$ and $27.75^{\circ} \mathrm{C}$; relative humidity oscillated between $79 \%$ and $82 \%$ (Fig. 1), and biomass reduction was $27.54 \%$ (Fig. 2). Adults and immature C. macellaria and C. albiceps were observed, while O. aenescens, Lasiophanes sp. and D. maculatus were found only as adults; L. eximia, M. domestica and Lepidodexia sp. were found in larval stage. It is important to notice that this stage was dominated by larvae of Calliphoridae (Tables II, III and IV).

Advanced (day 8 to 14). The carcass was characterized by reduction of flesh, preserving only the skin, cartilage, bones and remains of other tissues like intestines. Rectal body temperature varied between $30^{\circ} \mathrm{C}$ and $36.75^{\circ} \mathrm{C}$, while room temperature ranged between $23^{\circ} \mathrm{C}$ and $27.5^{\circ} \mathrm{C}$; relative humidity was $85 \%$ (Fig. 1). Due to the consumption of tissue mainly by larvae and dehydration, biomass loss was the highest $39.84 \%$ (Fig. 2). Immature and adults of C. macellaria, C. albiceps, Lepidodexia sp. dominated; M. domestica was found as larvae; D. maculatus and Lasiophanes sp. were also found in adult stage. The first pupae of $C$. macellaria were observed, as well as pupae and puparies of $C$. albiceps (Tables II, III and IV).

Remains (day 15 to 53). The carcass was reduced to skin, hairs and bones; its original form was not identifiable because the remains were dispersed due to degradation. Temperature oscillated between $26^{\circ} \mathrm{C}$ and $29.5^{\circ} \mathrm{C}$; relative humidity was more variable between $44 \%$ and $85 \%$ (Fig. 1), registering the lowest percentage, and biomass loss was $13.06 \%$ (Fig. 2). Larvae of D. maculatus were the markers of this decomposition stage, although adults of this species were also present, as well as of Lasiophanes sp., and closed and open puparies of C. albiceps (Tables II, III and IV).

Succession Matrix (Present-Absent). Due to the low diversity and high abundance of insects associated to the process, a matrix of ecological succession was built with the total number of species collected; Diptera: C. albiceps (larvae, pre-pupae, pupae, adult), C. macellaria (larvae, pupae, adult), L. eximia (larvae, adult), H. semidiaphana (adult), $M$. domestica (larvae), O. aenencens (adult), Oxysarcodexia sp. (adult), Lepidodexia sp. (larvae), Coleoptera: D. maculatus (larvae, adult) and Hymenoptera: Lasiophanes sp. (adult) (Table IV).

Species Accumulation Curve. Species increase during the first six days, time in which six out of the 10 species found appeared, reflected on the graphic by the steep slope; thereafter, species increase slowed down until day $20^{\text {th }}$ when total richness reached its maximum. The observed species corresponded to $100 \%$ of the expected species by Chao and Jack estimators, indicating that the sampling time was enough to find the maximum number of species associated to indoor body decay (Fig. 4).

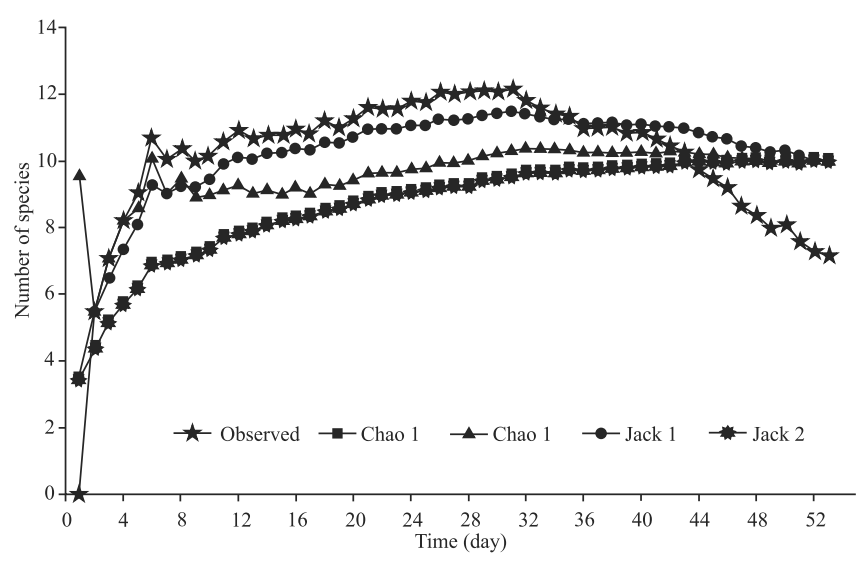

Fig. 4. Accumulation curve of species associated to indoor cadaveric decomposition in the Colombia Amazonia Piedmont. 
H(15000000000 $\pm \mid \begin{array}{llllllllll}0 & 0 & 0 & 0 & 0 & -0 & 0 & 0 & 0 & 0\end{array}$

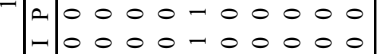
$\mid-1-000000-000$ $n$ صी 00000000000 $=\mid \begin{array}{llllllllll}n & 0 & 0 & 0 & 0 & -0 & 0 & 0 & 0 & 0\end{array}$ $1-00000-00000$ $|\varangle|-00000000$ N $\because \mid \begin{array}{lllllllllll}2 & 0 & 0 & 0 & 0 & -0 & 0 & 0 & 0 & 0\end{array}$ $1-0000-00000$

$-1<-0000000$

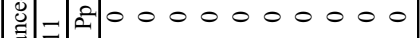
交 $\mid \begin{array}{lllllllllll}1 & 0 & 0 & 0 & 0 & -1 & 0 & 0 & 0 & 0 & 0 \\ - & 0 & 0 & 0 & 0 & -1 & 0 & 0 & 0 & 0 & 0\end{array}$ $1<00000-0-000$

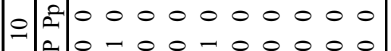

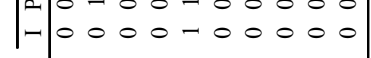

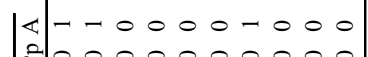
a की

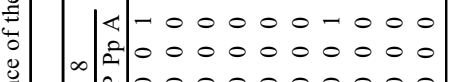
$\begin{array}{lllllllllll}0 & 0 & 0 & 0 & 0 & 0 & 0 & 0 & 0 & 0 \\ 0 & -1 & 0 & 0 & -1 & 0 & 0 & 0 & 0 & -1\end{array}$ 空 || $\begin{array}{llllllllll} & 0 & 0 & 0 & 0 & -0 & 0 & 0 & 0\end{array}$

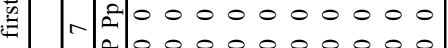
$\cong \quad \mid \begin{array}{lllllllllll}2 & 0 & 0 & 0 & 0 & 0 & 0 & 0 & 0 & 0 & 0 \\ 1 & 0 & -1 & - & 0 & -1 & 0 & 0 & 0 & 0 & -1\end{array}$ 苞

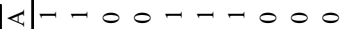
00000000000

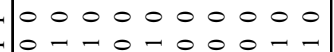
马 $\mid$ | $\mid-1-0$ ज़ी $+\quad \mid \begin{array}{lllllllllll}n & 0 & 0 & 0 & 0 & 0 & 0 & 0 & 0 & 0 & 0 \\ -1 & 0 & -1 & -1 & 0 & -1 & 0 & 0 & 0 & -1 & -1\end{array}$ $\ddot{\varangle}|-|-1-0-\cdots-0-0$

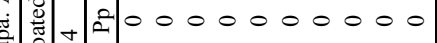

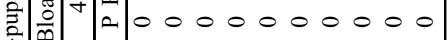
D.

\section{के}

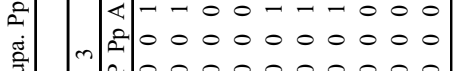
$\equiv \quad \mid \begin{array}{lllllllllll}2 & 0 & 0 & 0 & 0 & 0 & 0 & 0 & 0 & 0 & 0 \\ 1 & 0 & -1 & & 0 & 0 & 0 & 0 & 0 & 0 & 0\end{array}$ 窇 $\mid$ | $\mid$ |

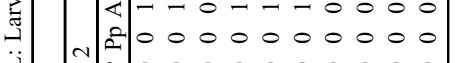

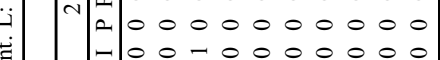
च $1-100-0000000$

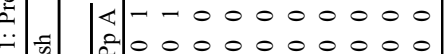

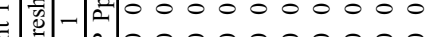
苟党 य $1-1-000000000000$

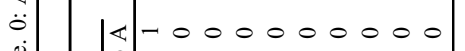

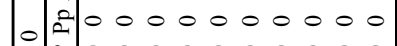
- 100000000000

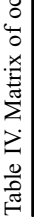

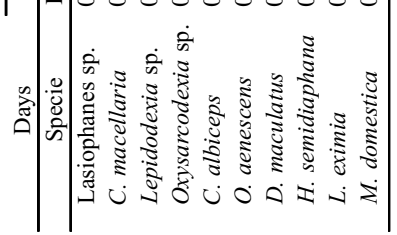

$\approx\left|\begin{array}{lllllllllll}\varangle \mid & 0 & 0 & 0 & 0 & 0 & - & 0 & 0 & 0 \\ 2 & 0 & 0 & 0 & 0 & -1 & 0 & 0 & 0 & 0 & 0 \\ 0 & 0 & 0 & 0 & 0 & - & 0 & 0 & 0 & 0 & 0\end{array}\right|$ $\begin{array}{lllllllllll}- & 0 & 0 & 0 & 0 & -1 & 0 & 0 & 0 & 0 & 0 \\ 0 & 0 & 0 & 0 & 0 & 0 & 0 & 0 & 0 & 0\end{array}$ $\varangle \mid-000000000$ iी $00000-00000$ $20000-00000$ 10000000000 $\mid \begin{array}{llllllllll}-1 & 0 & 0 & 0 & 0 & 0 & 0 & 0 & 0 & 0 \\ 0 & 0 & 0 & 0 & 0 & 0 & 0 & 0 & 0 & 0\end{array}$

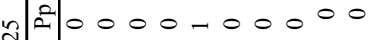
L $0000-00000$ $1-0000000000000$

$|<|-000-0-000$

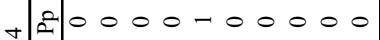
4 10000000000

$|\varangle|-0000000000$ ₹ी0 $0000-00000$ तิ $1-0000000000$ $|\varangle|-0000-0-0$

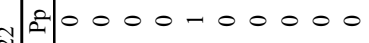
N10000 0000000 10000000000

$\mid-0$ - $000-00000$

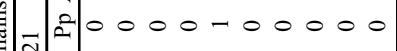
त $2000000-00000$ $-1000000000000$ $|<|-0 \quad 0 \quad 0-0-0,000$ 0 aी 000000 $\begin{array}{lllllllllll}2 & 0 & 0 & 0 & 0 & -0 & 0 & 0 & 0 & 0\end{array}$ $1-0000000000$ 《- -0 o $00-0-000$ 0 aी $000000-0$ $200000-00000$ $1-0000000000$

$|<|-0000-0-000$ $\infty$ 2ी0 $0000-00000$ $-1200000-00000$ 10000000000

$|<|-0000000$ Aे $00000-00000$ L $000000-00000$ $10000-00000$

$|-0|-000-00000$ 0 잉 $0000-0000$

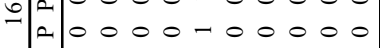
$10000-00000$

$|\ll| 0000-00000$

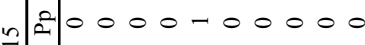
$20000-00000$

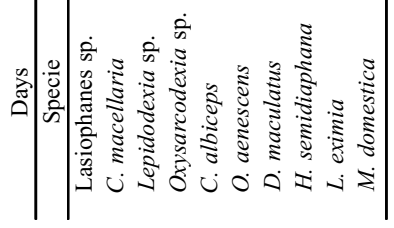

$|\varangle|-000000000$

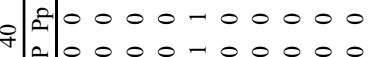
$1-000000-000$ $\mid-1-000000-000$

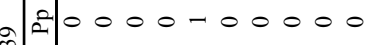
a $00000-00000$ $1000000-000$ $\mid<100000000000$ $\infty$ aे| 000000 ma $1000000-000$

$|<|-000000000$ $\sim$ Aे $00000-00000$ m $000000-00000$ 1- $000000-000$ $|<|-000000000$ صी $00000-00000$ $n=00000-00000$ - $000000-000$ $|<|-000-0-000$ 垔0 $0000-0000$ m $n=00000-00000$ $1000000-000$

$1<0000000-000$ + 青0 $000-00000$ m $a$ l $000000-00000$ $1-000000-000$

$|<|-0000000000$ $m$ 羊 $0000-0000$ ก L $1-000000-000$

$|<|-000000000$ N $2100000-0$ N $200000-00000$ $1000000-000$

$|<|-0000000$ - थे|l $0000-0000$ a $00000-00000$ $1-000000000$ $|<| 00000000000$ â|0 $0000-0000$ ก $1-10000000000$ $\mid-1-0000000000$ Aे $00000-0000$ N $200000-00000$ 10000000000

$\mid-1-00000-0$ $\infty$ حी $00000-0000$ $\sim$ N $000000-00000$ $1-1 \begin{array}{llllllllllll}0 & 0 & 0 & 0 & 0 & 0 & 0 & 0 & 0 & 0\end{array}$

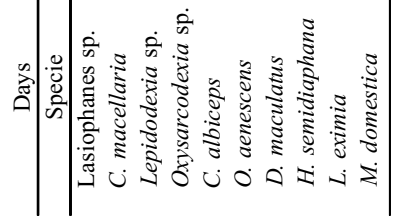

$|\varangle|-000000-0$ $n \mid \begin{array}{lllllllllll}2 & 0 & 0 & 0 & 0 & - & 0 & 0 & 0 & 0 & 0 \\ 0 & 0 & 0 & 0 & 0 & -1 & 0 & 0 & 0 & 0 & 0\end{array}$ $1-000000-000$

$\varangle-0000000$

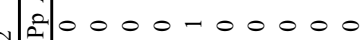
N $1-000000-000$

$1<10000000000$

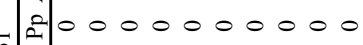
n $n$ a 000000000000 $1-1 \begin{array}{lllllllll}1 & 0 & 0 & 0 & 0 & 0 & -0 & 0 & 0\end{array}$

$|<|-0000000000$ - 2ิ0 $0000-00000$ $n$ a $-1000000-000$

$|<|-0000000000$ aी $0000-00000$

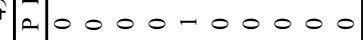
$-1000000-000$

$|<|-0000000000$

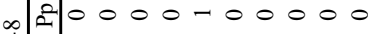

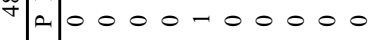
$1-00000-000$

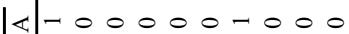
जी 0 a $00000-00000$ है $1-1000000-000$

《- -00000000 i) $00000-0000$ $10000-00000$ $-000000-000$ 《 $1-000000$ 蚟 $0000-00000$

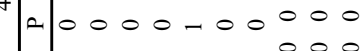
$-1000000-000$

$\ll-1-0000000$ मी $00000-0$ a $00000-00000$ $1-0000000$

| 1 - 000000000 $m$ चि $0000-00000$

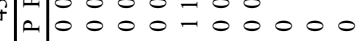
- $000000-000$

$1<-0000000$ 4 光 $00000-00000$ t $1-0 \begin{array}{llllllllll}0 & 0 & 0 & 0 & 0 & 0 & -0 & 0 & 0\end{array}$

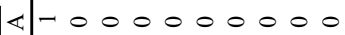
$=$ चे $0000-00000$ F $-10000000010$

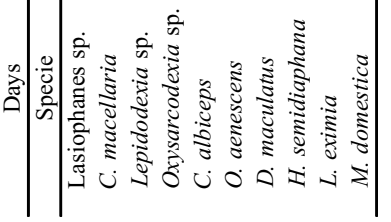

Revista Brasileira de Entomologia 58(4): 326-332, December 2014 


\section{DISCUSSION}

Five decaying stages were observed just like in other indoor studies (Centeno et al. 2002; Ahmad et al. 2011), the same number of stages observed in outdoor conditions (Mégnin 1894; Wolff et al. 2001; Vitta et al. 2007; Martínez et al. 2007; Ramos-Pastrana \& Wolff 2011).

In studies of decaying bodies outdoors, Smith (1986) affirmed that ovoposition may occur a few minutes after death; Fiedler et al. (2008) in study in western Germany about the diversity of Diptera on decomposition piglets on the edge forest and inside forest, reported the first eggs were deposited after a few minutes in the first and 30 minutes on the second situation during fresh decomposition; in Colombia, Wolff et al. (2001) observed the arrival of Diptera 30 minutes after slaughter and ovoposition during the bloated stage; Ramos-Pastrana \& Wolff (2011) reported the arrival of $C$. macellaria and $C$. albiceps under sunny conditions and of $L$. eximia and $H$. segmentaria under shadow conditions, respectively (4 and 7 minutes after death). In the present indoors study, the arrival of $C$. macellaria was registered on day 1 , similarly to the data obtained by Centeno et al. (2002), in which its arrival was registered on day 1 in the autumn, spring and summer, while on day 2 in winter; Ahmad et al. (2011) reported a 3-day delay in the arrival of colonizing species; other studies have also demonstrated such a delay (Goff 1991; Amendt et al. 2004).

In relation to total timing of the study, it was observed that in Colombian Amazon the time of decomposition from fresh to remains takes 54 days; while in Buenos Aires-Argentina in spring and summer 22 days, in winter and autumn 74 and 129 days respectively (Centeno et al. 2002).

From the bloated to the advanced stage, C. macellaria, $L$. eximia, C. albiceps, $M$. domestica and Lepidodexia sp. dominated tissue consumption. The Muscidae $O$. aenescens was present in greater abundance during all the decomposition process, in concordance with data obtained by Centeno et al. (2002), but contrasting with those of Ahmad et al. (2011), where the species of Chrysomya were dominant in these stages. Concerning the advanced decaying stage, larvae of D. maculatus were present, species reported also by Centeno et al. (2002), but without specifying its decaying stage. During the remains stage, high abundance of pupae of C. albiceps was found next to carcass.

Diptera was the prevalent order with more than $90 \%$ of the species, starting its activity from the fresh stage and reaching the highest number during the active stage, given by larval activity. Coleoptera and Hymenoptera were only 6\%; however, they were important components carrying out functions as necrophagous and predators, generating great dynamism throughout body decay. Studies of the succession of insects associated to decaying bodies in special microenvironments in Colombia are very significant for their contribution to the development of forensic science in the country, as well as a potential forensic tool in cases of human corpses found indoors.

\section{ACKNOWLEDGMENTS}

Thanks to the entomology seed research of University of the Amazonia; to Colciencias Project 601-2009 (YR); to Universidad de Antioquia; To Dr. César A. Estrada González (in memorian), research Vice Dean at the University of Amazonia; to Jhoha E. Cárdenas García, Jesús Matiz Caicedo, Yenny Rojas Grande, Cindy K. Andrade, Cesar Valverde and Yesion J. Penagos García; MSc. Sandra Pérez; to biology student Carlos Andrés Londoño.

\section{REFERENCES}

Ahmad, N.W., Lim, L.H., Dhang, C.C., Chin, H.C., Abdullah, A.G., Mustaffa, W.-N.W., Kian, C.W., Jeffery, J., Hanshim, R. \& Azirum, S.M. 2011. Comparative insect fauna succession on indoor and outdoor monkey carrion in a semi-forested area in Malaysia. Asian Pacific Journal of Tropical Biomedicine 2: S232 S238.

Almeida, L.M. \& Mise, K.M. 2009. Diagnosis and key of the main families and species of South America Coleoptera of Forensic importance. Revista Brasileira de Entomologia 53: 227244.

Amat, E., Vélez, M.C. \& Wolff M. 2008. Clave ilustrada para la identificación de los géneros y las especies de callifóridos (Diptera: Calliphoridae) de Colombia. Revista Caldasia 30: 231244.

Amendt, J., Krettek, R. \& Zehner, R. 2004. Forensic entomology. Naturwissenschaften 91: 5165.

Anderson, G.S. \& VanLaerhoven, S.L. 1996. Initial studies on insect succession on carrion in southwestern British Columbia. Journal of Forensic Science 41: 617-625.

Anderson, G.S. 2010. Factors that influence insect succession on carrion, p. 201 250. In: Byrd, J.H. \& Castner, J.L. (Orgs.). Forensic Entomology: The Utility of Arthropods in Legal Investigations. 2nd Ed. CRC Press, xxiii+708 p.

Arnaldos, I., Romera, E., Garcia, M.D. \& Luna, A. 2001. An initial study on the succession of sarcosaprophagous Diptera (Insecta) on carrion in the southeastern Iberian Peninsula. International Journal of Legal Medicine 114: 156162.

Barrios, M. \& Wolff, M. 2011. Initial study of arthropods succession and pig carrion decomposition in two freshwater ecosystems in the Colombian Andes. Forensic Science International 212: 164-172.

Benecke, M. 1998. Six forensic entomology cases: Description and commentary. Journal of Forensic Sciences 43: 797805.

Benecke, M. 2008. A brief survey of the history of forensic entomology. Acta Biologica Benrodis 14: 1538 .

Brown, B.V., Borkent, A., Cumming, J.M., Wood, D.M., Woodley, M. \& Zumbado, M.A. 2009. Manual of Central American Diptera. Vol. 1. Ottawa, NRC Research Press, $x i+714$ p.

Brown, B.V., Borkent, A., Cumming, J.M., Wood, D.M., Woodley, M. \& Zumbado, M.A. 2010. Manual of Central American Diptera. Vol. 2. Ottawa, NRC Research Press, $x v+728$ p.

Camacho, G. 2005. Sucesión de la entomofauna cadavérica y ciclo vital de Calliphora vicina (Diptera: Calliphoridae) como primera especie colonizadora, utilizando cerdo blanco (Sus scrofa) en Bogotá. Revista Colombiana de Entomología 31: 189197.

Carvalho, C.J.B. \& Ribeiro, P.B. 2000. Chave de identificação das espécies de Calliphoridae (Diptera) do sul do Brasil. Revista Brasileira de Parasitologia Veterinária 9: 169173.

Carvalho, L.M.L, Thysen, P.J., Linhares, A.X. \& Palhares, F.A.B. 2000. A checklist of arthropods associated with pig carrion and human corpses in southeastern Brazil. Memórias do Instituto Oswaldo Cruz 95: 135138.

Carvalho, L.M.L. \& Linhares, A.X. 2001. Seasonality of insect succession and pig carcass decomposition in a natural forest area in southeastern Brazil. Journal of Forensic Science 46: 604608.

Carvalho, C.J.B., Rafael, J.A., Souto, M. \& Silva, V.C. 2012. Diptera, p. 702 744. In: Rafael, J.A., Melo, G.A.R., Carvalho, C.J.B., Casari, 
S.A. \& Constantino, R. (eds.). Insetos do Brasil, Diversidade e Taxonomia. Ribeirão Preto, Holos Editora, xiv +810 p.

Centeno, N., Maldonado, M. \& Oliva, A. 2002. Seasonal patterns of arthropods occurring on sheltered and unsheltered pig carcasses in Buenos Aires Province (Argentina). Forensic Science International 126: 6370.

Colwell, R.K. 2006. EstimateS: Statistical estimation of species richness and shared species from samples (Ver. 8). Available at: http:// purl.oclc.org/estimates (accessed 15 December 2012).

Cruz, T.M. \& Vasconcelos, S.D. 2006. Entomofauna de solo associada à decomposicão de carcaça de suíno em um fragmento de Mata Atlântica de Pernambuco, Brasil. Biociências 14: 193-201.

De Jong, G., \& Hoback, W. 2006. Effect of investigator disturbance in experimental forensic entomology: succession and community composition. Medical Veterinary Entomology 20: 248-258.

Fiedler, A., Halbach, M., Sinclair, B. \& Benecke, M. 2008. What is the edge of a forest? A diversity analysis of adult of Diptera found on decomposition piglets inside and on the edge of Western German woodland inspired by a courtroom question. Entomologie Beute 20: 173-191.

Florez, E. \& Wolff, M. 2009. Descripción y clave de los estadios inmaduros de las principales especies de Calliphoridae (Diptera) de importancia forense en Colombia. Neotropical Entomology 38: 418429.

Frost, C.L., Braig, H.R., Amendt, J. \& Perotti, M.A. 2010. Indoor arthropods of forensic importance: Insects associated with indoor decomposition and mites as indoor markers, p. 93 108. In: Amendt, J., Campobasso, C.P., Goff, M.L. \& Grassberger, M. (eds.). Current concepts in forensic entomology. Dordrecht, Springer, viii+376 p.

Grassberger, M. \& Frank, C. 2004. Initial study of arthropod succession on pig carrion in central European urban habitat. Journal of Medical Entomology 41: 511523.

Gennard, D.E. 2007. Forensic Entomology. An Introduction. John Wiley \& Sons Ltd., $x i x+254$ p.

Goff, M.L. 1991. Comparison of insect species associated with decomposing remains recovered inside dwellings and outdoors on the island of Oahu, Hawaii. Journal of Forensic Sciences 36: 748753.

Grisales, D., Ruiz, M. \& Villegas, S. 2010. Insects associated with exposed decomposing bodies in the Colombian Andean Coffee Region. Revista Brasileira de Entomologia 54: 637644.

Hall, M., Brown, T., Jones, P. \& Clarck, D. 2008. Forensic sciences, p. 463 496. In: Cox, M., Flavel, A., Hanson, I., Laver, J. \& Wessling, R. (eds.). The scientific investigation of mass graves: Towards protocols and standard operating procedures. Cambridge, Cambridge University Press, xxviii $+592 \mathrm{p}$.

Hall, R.D. \& Huntington, T.E. 2010. Introduction: Perceptions and Status of Forensic Entomology, p. 1-16. In: Byrd, J.H. \& Castner, J.L. (eds.). Forensic Entomology: The Utility of Arthropods in Legal Investigations. 2nd Ed. CRC Press, xxiii+708 p.

Haskell, N.H. 1990. Entomological collection techniques at autopsy and for specific environments, p. 98 110. In: Catts, E.P. \& Haskell, N.H. (eds.). Entomology \& Death: A Procedural Guide. Joyce's Print Shop, Inc., xii+182 p.

Iannacone, J. 2003. Artropofauna de importancia forense en un cadáver de cerdo en el Callao, Perú. Revista Brasileira de Zoologia 20: 8590.

I.G.A.C. 2010. Caquetá. Características Geográficas. Instituto Geográfico Agustín Codazzi, Subdirección de Geografía y Cartogafia, 376 p.

Jiménez-Valverde, A. \& Hortal, J. 2003. Las curvas de acumulación de especies y la necesidad de evaluar la calidad de los inventarios biológicos. Revista Ibérica de Aracnología 8: 151161.

Jirón, L.F., Vargas, L.G., Vargas-Alvarado, E. 1982. Four muscoid flies (Sarcophagidae and Muscidae) associated with human cadavers in Costa Rica. Brenesia 21: 15.

Magaña, C. 2001. La entomología forense y su aplicación a la medicina legal. Data de la muerte. Boletín de la Sociedad Entomológica Aragonesa 28: 49161.

Mann, R.W., Bass, W.M. \& Meadows, L. 1990. Time since death and decomposition of the human body: Variables and observations in case and experimental field studies. Journal of Forensic Sciences 35: 103111.

Mariluis, J.C. \& Peris, S.V. 1984. Datos para una sinopsis de los Calliphoridae neotropicales. Revista Española de Entomología 60: 6786.
Martínez, E., Duque, P. \& Wolff, M. 2007. Succession pattern of carrionfeeding insects in Paramo, Colombia. Forensic Science International 166: 182189

Mégnin, P. 1894. La faune de cadavres. Application de l'entomologie a la médicine légale. Encyclopédie Scientifique des Aides-Mémorie. Paris, Masson \& Gauthier-Villars, 214 p.

Moura, M.O., Carvalho C.J.B. \& Monteiro-Filho. 1997. A preliminary analisis of insects of medico-legal importance in Curitiba, state of Paraná. Memórias do Instituto Oswaldo Cruz 92: 269274.

Palacio, E.E. \& Fernández, F. 2003. Clave para las Subfamilias y Géneros, p. 233 260. In: Fernández, F. (ed.). Introducción a las hormigas de la región Neotropical. Bogotá, Instituto de Investigación de Recursos Biológicos Alexander von Humboldt, xxvi+398 p.

Peris, S. 1992. A preliminary key to the world genera of the subfamilies Toxotarsinae, Chrysomyinae and Rihiinae (Diptera, Calliphoridae). Boletín de la Real Sociedad Española de Historia Natural, Seccion Biología 88: 7998.

Pérez, S., Duque, P. \& Wolff, M. 2005. Successional behavior occurrence matrix of carrion-associated arthropods in the urban area of Medellin, Colombia. Journal of Forensic Science 50: 448-454.

Piatkowski, S. 1991. Synanthropic flies in an 11-story apartment house in Gdañsk. Wiad Parazytologie 37: 115117.

Pohjoismäki, J.L.O., Karhunen, P.J., Goebeler, S., Sauko, P. \& Sääksjärvi, I.E. 2010. Indoors forensic entomology: Colonization of human remains in closed environments by specific species of sarcosaprophagous flies. Forensic Science International 199: 3842.

Queiroz, S.M.P. \& Carvalho, C.J.B. 1987. Chave pictórica e descrições de larvas de 3o. instar de Diptera (Calliphoridae, Muscidae e Fanniidae) em vazadouros de resíduos sólidos domésticos em Curitiba, Paraná. Anais da Sociedade Entomológica do Brasil 16: 265288.

Ramos-Pastrana, Y. \& Wolff, M. 2011. Entomofauna cadavérica asociada a cerdos expuestos al sol y sombra, en el Piedemonte Amazónico Colombiano. Momentos de Ciencia 8: 4554.

Ribeiro, P.B. \& Carvalho, C.J.B. 1998. Pictorial key to Calliphoridae genera (Diptera) in southern Brazil. Revista Brasilera de Parasitologia Veterinária 7: 137140 .

Schroeder, H., Klotzbach, H., Oesterhelweg, L. \& Püschel, K. 2002. Larder beetles (Coleoptera, Dermestidae) as an accelerating factor for decomposition of a human corpse. Forensic Science International 127: 231236.

Segura, N.A., Usaquén, W., Sánchez, M.C., Chuaire, L. \& Bello, F. 2009. Succession pattern of cadaverous entomofauna in a semi-rural area of Bogotá, Colombia. Forensic Science International 187: 6672.

Segura, N.A., Bonilla, M.A., Usaquén, W. \& Bello, F. 2011. Entomofauna resource distribution associated with pig cadavers in Bogotá DC. Medical and Veterinary Entomology 25: 4652.

Smith, K.G.V. 1986. A Manual of Forensic Entomology. London, Department of Entomology, British Museum (Natural History), 137 p.

Souza, A.S.B., Kirst, F.D. \& Kruger, R.F. 2008. Insects of forensic importance from Rio Grande do Sul State in southern Brazil. Revista Brasileira de Entomologia 52: 641646.

Tabor, K., Fell, R. \& Brewster, C. 2005. Insect fauna visiting carrion in Southwest Virginia. Forensic Science International 150: 7380.

Vasconcelos, S.D. \& Araujo, M.C.S. 2012. Necrophagous species of Diptera and Coleoptera in northeastern Brazil: state of the art and challenges for the Forensic Entomologist. Revista Brasileira de Entomologia 56: 714.

Velásquez, Y. 2008. A checklist of arthropods associated with rat carrion in a mountain locality of northern Venezuela. Forensic Science International 174: 6769

Vitta, A., Pumidonming, W., Tangchaisriya, U., Poodendean, C., \& Nateeworanart, S. 2007. A preliminary study on insects associated with pig (Sus scrofa) carcasses in Phitsanulok, northern Thailand. Tropical Biomedicine 24: 15.

Wolff, M., Uribe, A., Ortiz, A. \& Duque, P. 2001. A preliminary study of Forensic Entomology in Medellín, Colombia. Forensic Science International 120: 5359.

Received 30 May 2014; accepted 9 September 2014

Associate Editor: Rodrigo F. Krüger 